ISBN 978-981-11-3671-9

Proceedings of 2017 the 7th International Workshop on Computer Science and Engineering

(WCSE 2017)

Beijing, 25-27 June, 2017, pp. 1324 -1331

doi: $10.18178 /$ wcse.2017.06.230

\title{
A Universal Interface for Programming Electronic Shelf Labels in Retailing Contexts
}

\author{
I-Ching Chen ${ }^{1}$ and Shueh-Cheng $\mathrm{Hu}^{2+}$ \\ ${ }^{1}$ Department of Information Management Chung Chou University of Science and Technology Chang Hua 51003, \\ Taiwan, ROC \\ ${ }^{2}$ Department of Computer Science \& Comm. Engineering Providence University Taichung 43301, Taiwan, ROC
}

\begin{abstract}
In contrast to traditional paper price tags, electronic shelf label (ESL) has made dynamic pricing feasible and efficient in brick-and-mortar retailers. Even better, ESLs enabling two-way communication with shoppers in front of shelf are able to realize some innovative business strategies, with involvement of merchants, shoppers, advertising agents, and other business partners. However, to make ESLs programmable and easily integrable by heterogeneous information systems, a universal interface is necessary. This article examines various application scenarios of ESLs in retailing contexts and accordingly proposes a universal interface for programming these ESLs.
\end{abstract}

Keywords: Electronic shelf label; interactive IOT object; restful interface; retailers.

\section{Introduction}

In retailers, many goods' prices need to be updated frequently to reflect dynamic factors such as inventory level, prices of competitors, expiration date, etc. Obviously, brick-and-mortar retailers cannot update their paperbased price tags being attached on the shelf automatically. Electronic shelf label (ESL), is also known as electronic price tag or digital price tag, emerges as an alternative for displaying prices and other product information in brick-and-mortar retailers. In contrast to paper-based tags, what shown on ESLs including prices and other product information could be dynamically changed by retailers' inventory information system, which means large scale pricing dynamics is feasible and efficient in physical retail stores. With the capability of dynamically changing prices, physical retailers have better chance to compete with their online counterparts. Up to date, more than hundreds of millions of ESLs have been deployed in food retailers, consumer electronics stores, supermarkets, pharmacy, and other sectors. Even though, the ESL's penetration rate in global retail market is still pretty low. Figure 1 shows samples of ESLs and an application scenario in a grocery store.

Not only displaying dynamic information including prices, promotions, smart ESLs further enable two-way communication between ELSs (or the backend information systems) and shoppers in front of shelf. Moreover, with application of mobile devices and APPs, personalized shopping experience could be brought to physical retailing contexts from online stores. The information dynamics, two-way communication, and personalization

\footnotetext{
+ Corresponding author. Tel.: +886-922-937-037.

E-mail address: jineandya@gmail.com; shuehcheng@gmail.com
} 
collectively are able to realize versatile business strategies that promote sales, reduce waste, elevate store image, strengthen customer loyalty, and so on. That will provide retail businesses, especially those retailers with multichannels, competitive edges.

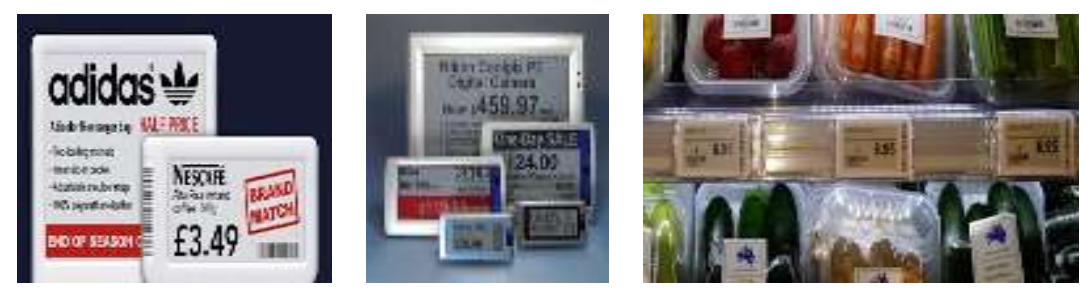

Fig. 1: Electronic shelf labels and applicaion

However, to fully exploit the edges of ESLs in enterprise-wide retailing contexts, the application of ESLs must be considered beyond a single store's viewpoint. Technically speaking, all deployed ESLs could be accessible by not only retailers, but also shoppers, advertising agents, suppliers, and other business partners. In other words, ELSs could be treated as a kind of objects in the IOT (Internet of things), and various information systems being controlled by different parties need to access this kind of objects to fulfill particular functions.

In light of its significance in efficiently operating and managing a modern retail business, the present work aims to analyze the functions of ESLs in retailing contexts, and propose a universal interface for managing them. The universal interface, is easily accessible not only by merchants, but also by customers, business partners, and will provide business partners more flexibility to innovate and adjust collective business strategies, which are crucial in business contexts facing fierce competition. Besides, the universal interface facilitates seamless integrations of heterogeneous information systems in different parties.

\section{Prior Works Review}

\subsection{Electronic shelf labels}

Prior studies show that price is one of the most significant factors influencing customers' purchase intention and behaviors in retailing contexts [1-3]. Online retailers can update prices and other product information promptly and economically, through their back end information systems. The pricing dynamics provide them superior flexibility and adaptability, comparing with their brick-and-mortar counterparts. Facing increasing competition from online retailers, many traditional retailers shrank or retreated from the market since the inception of e-commerce $[4,5]$.

In a physical retailer selling goods with frequent price fluctuations such as supermarkets, grocery stores, and consumer electronics retailers, staff must manually change price tags from time to time. In a large scale supermarket, thousands of price tags must be changed per week. The manual tags' changing results in the following 3 drawbacks: (1) slow response, which means prompt and volume price changes are difficult if not impossible. (2) Labor consuming, which means higher operation cost. And (3) price discrepancy, which means the price shown on tag is different from the one in information system and will be charged to shoppers. The price discrepancy further creates adverse effect on store image and customer loyalty [6-8].

To cope with this troublesome but significant issue, retailers gradually adopt electronic shelf labels, even at high initial cost. Although an ESL can show information with E-Ink, LCD, or other display technology, but most of deployed ESLs took E-Ink due to its superior power efficiency; one button cell battery can last for 5 years in normal usage scenario. In the context of retailing, higher power efficiency of large volume electronic price tags 
means much less labor-intensive works and lower operation and maintenance cost. The major suppliers in the ESL market include SES-imagotag [9], Pricer [10], Opticon [11], Altierre [12], and Hanshow [13].

Figure 2 shows a modern retailing context with deployment of ESLs and supportive devices. A product information server updates the product information including price, updated product information will be sent to corresponding ESLs through gateway and wireless network. With this kind of deployment, prices of thousands of products could be updated within seconds.

To make ESLs more versatile, QR codes were shown on ESLs, so that shoppers in front of shelves can interact with merchant's information systems to acquire more relevant information such as product review, rating, usage, etc. In addition, near field communication (NFC) capability was added to some ESLs, which makes interactions between shoppers and retailing contexts more convenient [14].

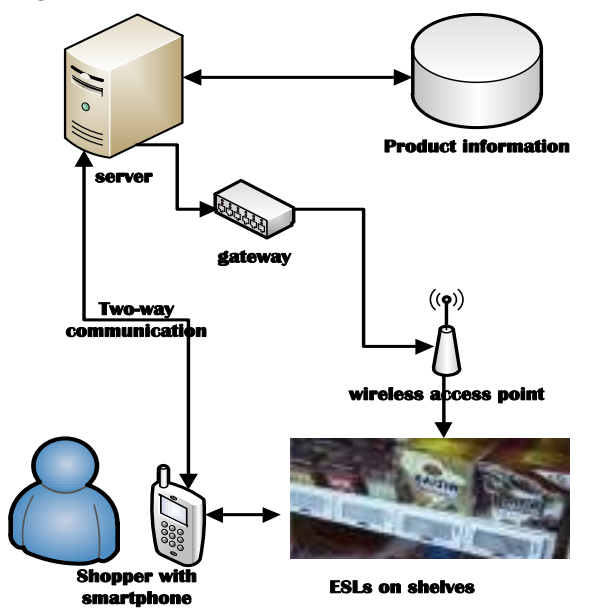

Fig. 2: Retailing Context with ESLs

\subsection{IOT and restful styled interface}

With connections to back end information systems via wireless networks, each ESL could be indirectly accessible by specific computers on the Internet. From this viewpoint, ESLs within retailing contexts are a kind of IOT (internet of things) objects. A prior work describes how to plan and deploy ESL enabled networks in details, but within one single store's scope [15].

To make IOT objects programmable (manageable) by various information systems, an application programming interface (API) that fits different types of systems is necessary. Prior works indicated that RESTful [16] styled interface is appropriate for programming different types of IOT objects [17-20].

In a RESTful application, everything such as ESLs that could be accessed or operated is treated as resources, and resources must be identifiable via a uniform naming scheme, or practically, the uniform resource identifier (URI). In contrast with its heavyweight counterpart: the SOAP-based Web service, the restful service associates standard HTTP methods with operations that intended to be performed on resources.

Due to the uniform identifying scheme and access (operation) interface, the restful approach significantly reduces the complexity that are caused by processing of SOAP-encoded messages, such reduction offers a lightweight option for adopting Web services. Moreover, the plain HTTP-based access interface eases the integration of popular and lightweight techniques such as asynchronous JavaScript and XML (AJAX) into client side of restful Web applications, which further makes more service consumers using devices with various form factors to access IOT resources pervasively. In addition, applications supporting RESTful Web Services perform better on wireless nodes with constrained resources just like ESLs [20]. 
Considering many advantages of RESTful styled interface, this work realized a universal interface of ESLs with the RESTful style and the supported techniques.

\section{Application Scenarios of ELS}

Before proposing how to design the programmable interface for ESLs, this section analyzes several key application scenarios of ESLs in retailers.

\subsection{Dynamic pricing}

The most straightforward advantage of adopting ESLs in physical retailers is pricing dynamics. Many kinds of information influencing prices could be collected from in-store and external systems, after calculations according to pricing strategies, prices and other product information will be sent to and displayed on the corresponding physical ELSs. Information systems need to perform this kind of updating operations on ESLs frequently. Thus, performing these operations need to be very efficient.

\subsection{Product information online inquiry}

With accessible and networked ESLs, shoppers can obtain prices and other information regarding products of interest via computers or smartphones. In other words, shoppers can check prices of products in physical stores without visiting stores in person. Offering this function, brick-and-mortar retailers make their prices transparent to potential consumers who browse before purchasing, online price comparison agents or robots via which many shoppers collect candidate products. Clearly, this feature can pull physical stores back on many consumers' radar screen. This kind of reading (fetching) operations should be the most active ones, among all possible operations on the ESLs.

\subsection{Personalized experience}

Since increasing smartphones equip the NFC to realize convenient functions such as mobile payment, vendors can apply the same technology in ESLs to enable two-way communication between ESLs (the information system directly controls the ESLs) and shoppers (shopper's mobile APP).

Shoppers who use smartphones with personal identity can even obtain personalized product and sales information while interacting with NFC-enabled ESLs. For example, members of loyalty program who used smartphone to tap particular ESLs will be recognized and get special discount, or will obtain personalized recommendations and promotions messages via mobile APPs, according to her/his purchase history, preference, etc. This personalized shopping experience not only fits shoppers' needs well, but also will make vendors' marketing activities more prompt and precise.

\subsection{Product adjustment}

Retailers need to add and remove products from time to time, according to availability, season, market trend, and many other factors. Consequently, ESLs associated with added and removed products also need to be adjusted accordingly. Systems perform create and delete operations on particular ESL objects to adjust product alignment.

\section{Realizing a Restful Interface of ESLs}

This section explains the design rationales of the restful interface of programming ESLs, as well as describes how the design concepts were realized accordingly.

\subsection{Design rationales}


To embody the concept of universal programmable ESLs, a corresponding system was designed and implemented. The system consisting of major components is illustrated in figure 3 . The design of the interface complied with the following constraints: first, all types of ESLs will be organized in hierarchical style. For example, a chain retailer's ESLs consist of every individual store's ESLs that in turn comprising ESLs associated with different categories of products.

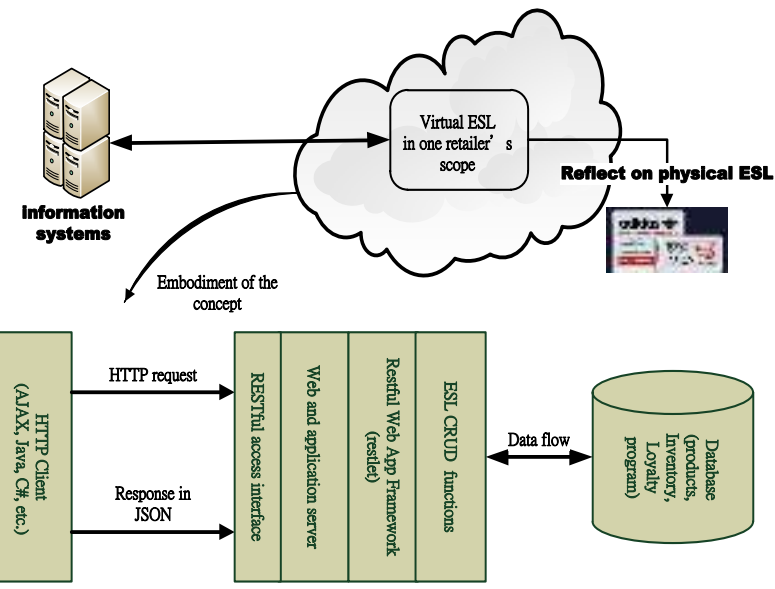

Fig. 3: Universal Programmable ESLs

Second, from the perspective of chain store's administrator, to make each product manageable in different levels, identifiers of all ESLs must follow a common URI pattern, and thus could be represented hierarchically. For example, to identify a particular product's ESL (say, with SKU =1001) in a specific store, we can use URL like this: http://www.allfoods.com /southernLA /store10/vege/SKU1001.

Third, the four basic HTTP methods: POST, GET, PUT, and DELETE are uniformly used to map the CRUD (Create, Read, Update, and Delete) operations on a virtual ESL, respectively. Accordingly, to create a new instance of virtual ESL record corresponding to a particular product, a POST request including the HTTP verb along with the request contents (input data) would be sent to http://www.allfoods.com/southernLA/store10/vege/. To update information of a particular product, the corresponding HTTP PUT request and the new data will be sent to http://www.allfoods.com/southernLA /store10 /vege/SKU1001.

\subsection{Implementation}

To realize the design concept, as figure 3 illustrates, each ESL need to be associated with a virtual ESL in retailer's information system. The virtual ESL records all information regarding one particular physical ESL that should not be exposed directly to external systems for reasons like security and power efficiency. All operations including create, retrieve, update, and delete are performed on the virtual ESL first, which status then be forwarded to the physical counterpart on demand.

A Web and application server was used to accept requests from clients; the restlet package was used for frameworking the restful Web application with Java servlet and JSP techniques. To persist all data records regarding contents in retailers' portfolios, relational database was the choice. On the client side of the proof-ofconcept system, the AJAX was applied to send the four basic HTTP requests, as well as to receive and handle the response messages from the service provider. A typical restful application allows that resources have different formats, e.g. plain text, XML, and JSON (JavaScript Object Notation), etc.

\section{Discussion and Conclusions}




\subsection{Smart ESLs and impacts}

The networked and interactive ESLs bring 3 major implications to administrators and users as follows:

1) Sensibility: online stores use cookies to keep track of online shoppers' purchasing behaviors completely and precisely. Smart ESLs enabling two-way communications behave to certain extent like sensory organs in brick-and-mortar retailer. Using interactive ESLs and mobile APPs, physical retailers also can know and record shoppers' behaviors for providing personalized services and conducting precise marketing. Collections of individual shoppers' behaviors are valuable to vendors.

2) Pervasiveness: fundamentally, the RESTful ESL objects could be accessed through basic HTTP methods. Consequently, the widely support of HTTP client makes it feasible to perform ESLs' functions diversely and pervasively. This is a critical feature making the ESLs-based system be adaptive to the ever-changing business strategies and contexts.

Besides the pervasiveness, the simple application interface utilizing URI and HTTP methods also reduces work load of systems managing ESLs significantly because of the lower time complexity of algorithms that are responsible for composing and decomposing large volume of messages between clients and ESL managers. Obviously, the simplicity of the RESTful interface benefit users of mobile devices in particular due to the inferior computing power.

3) Accessibility: realizing many business strategies needs seamless collaboration among multiple participants accessing ESLs. For example, analyzing customers' interests on a particular product need to consolidate data from many stores located on different cities. Fortunately, the common and standardized RESTful interface makes it easier to consolidate data from distributed ESLs. Actually, it is straightforward to access ESL-typed objects and integrate them directly within any type of HTTP client including the application of AJAX technique in most Web browsers.

\subsection{Contribution}

This article describes a RESTful styled approach for programming distributed ESLs that spread across enterprise-wide range. With the universal and simplicity aspects, heterogeneous information systems that reside on different sites and fulfill different functions, can integrate large volume of ESLs into current application frameworks with ease. Besides, users including shoppers, staff and managers, partners can use various connected client devices to conveniently access ESLs of interest.

\subsection{Future directions}

Since the application of ESLs in physical retail contexts is still in its very early stage, many innovative business strategies using ESLs are under development or will be developed and tested later. Rationally, effective and cost-efficient usage models of ESLs and the corresponding technical requirements are still evolving, which means the present programmable interface of ESLs might also adapt to the new approaches. Even though, the simplicity and universal properties of the proposed interface will prevail due to the features of ESL-enabled retailing contexts.

\section{Acknowledgment}

This research work has being funded by the grant from the Ministry of Science and Technology, Taiwan, ROC, under Grant No. MOST 104-2410-H-235-002. We deeply appreciate their financial support and encouragement.

\section{References}


[1] B. L. Alford and A. Biswas, "The effects of discount level, price consciousness and sale proneness on consumers' price perception and behavioral intention," Journal of Business research, vol. 55, pp. 775-783, 2002.

[2] M. Moore and J. Carpenter, "The effect of price as a marketplace cue on retail patronage," Journal of Product \& Brand Management, vol. 15, pp. 265-271, 2006.

[3] J. Lauren Munger and D. Grewal, "The effects of alternative price promotional methods on consumers' product evaluations and purchase intentions," Journal of Product \& Brand Management, vol. 10, pp. 185-197, 2001.

[4] J. Somaney. (2016, Feb 8). October Retail Sales Report Spells Trouble For Brick-And-Mortar, Shows Optimism For Amazon. Available: http://www.forbes.com/sites/jaysomaney/2016/11/16/october-retail-sales-report-spells-troublefor-brick-and-mortar-shows-optimism-for-amazon/\#436423aae18b

[5] E. Tinsley. (2017, Feb 9). Traditional brick-and-mortar stores closing, online retailers opening real stores. Available: http://www.9news.com/money/business/traditional-brick-and-mortar-stores-closing-online-retailers-opening-brickand-mortar-stores/384576414

[6] L. Xia, K. B. Monroe, and J. L. Cox, "The price is unfair! A conceptual framework of price fairness perceptions," Journal of marketing, vol. 68, pp. 1-15, 2004.

[7] J. E. Urbany, W. O. Bearden, A. Kaicker, and M. Smith-de Borrero, "Transaction utility effects when quality is uncertain," Journal of the Academy of Marketing Science, vol. 25, p. 45, 1997.

[8] V. Kumar, M. Hurley, K. Karande, and W. J. Reinartz, "The impact of internal and external reference prices on brand choice: the moderating role of contextual variables," Journal of Retailing, vol. 74, pp. 401-426, 1998.

[9] SES-imagotag. (2017, Feb 3). A strategic weapon for retailers, SES-imagotag's electronic shelf labels (ESLs). Available: http://www.ses-imagotag.com/en/products/\#electronic-shelf-labels

[10] Pricer. (2017, Jan 29). Why choose electronic shelf labels? Available: http://www.pricer.com/en/Benefits-ofElectronic-Shelf-Labels/

[11] Opticon. (2017, Jan 29). Opticon Display Solutions: a new in-store shopping experience. Available: http://opticon.com/product-category/display-solutions/esl/

[12] Altierre. (2017, Jan 9). The Altierre e-Paper labels, benefit from exceptional contrast and legibility thanks to their high-resolution display! Available: http://www.altierre.com/wordpress/index.php/solution/lcd-e-paper-tags/ep-tagfamily/

[13] Hanshow. (2017, Jan 9). What Happens When ESL Technologies Enter Retail? Available: http://www.hanshow.com/esl-retail-solution/

[14] C.-C. Teng, M. Bishop, J. Brown, A. Hansen, R. Kleinman, and N. Palmer, "Personalized shopping experience with NFC smartphone apps and electronic shelf label," in Proc. The Sixth International Conference on Advances in Human oriented and Personalized Mechanisms, Technologies, and Services. Venice, Italy, 2013, pp. 7-11.

[15] J. Sung, "End of paper labels: Emerging smart labels toward Internet of Things," in 2015 IEEE 2nd World Forum on Internet of Things (WF-IoT), 2015, pp. 216-221.

[16] R. T. Fielding, "Architectural Styles and the Design of Network-based Software Architectures," DOCTOR OF PHILOSOPHY, Information and Computer Science, UNIVERSITY OF CALIFORNIA, IRVINE, Irvine, California, 2000.

[17] M. Laine, "Restful web services for the internet of things," Online]. Saatavilla: http://media. tkk. fi/webservices/personnel/markku_laine/restful_web_services_for_the_internet_of_things.pdf, 2012.

[18] B. Xu, L. Da Xu, H. Cai, C. Xie, J. Hu, and F. Bu, "Ubiquitous data accessing method in IoT-based information system for emergency medical services," IEEE Transactions on Industrial Informatics, vol. 10, pp. 1578-1586, 2014. 
[19] M. Kovatsch, M. Lanter, and S. Duquennoy, "Actinium: A restful runtime container for scriptable internet of things applications," in Internet of Things (IOT), 2012 3rd International Conference on the, 2012, pp. 135-142.

[20] T. Yashiro, S. Kobayashi, N. Koshizuka, and K. Sakamura, "An Internet of Things (IoT) architecture for embedded appliances," in Humanitarian Technology Conference (R10-HTC), 2013 IEEE Region 10, 2013, pp. 314-319. 